\title{
Les Franco-Mauriciens : une diaspora pollinisée
}

\author{
Catherine Boudet
}

\section{OpenEdition}

Journals

Édition électronique

URL : https://journals.openedition.org/remi/4215

DOl : $10.4000 /$ remi.4215

ISSN : $1777-5418$

Éditeur

Université de Poitiers

\section{Édition imprimée}

Date de publication : 1 décembre 2007

Pagination : 109-132

ISBN : 978-2-911627-47-4

ISSN : 0765-0752

Référence électronique

Catherine Boudet, «Les Franco-Mauriciens : une diaspora pollinisée », Revue européenne des migrations internationales [En ligne], vol. 23 - n³ | 2007, mis en ligne le 01 décembre 2010, consulté le 14 avril 2022. URL : http://journals.openedition.org/remi/4215; DOI : https://doi.org/10.4000/remi. 4215

Ce document a été généré automatiquement le 14 avril 2022.

(c) Université de Poitiers 


\title{
Les Franco-Mauriciens : une diaspora pollinisée
}

\author{
Catherine Boudet
}

Le concept de diaspora est généralement utilisé pour exprimer la dispersion de populations politiquement minoritaires ou d'élites économiques. La diaspora évoque ainsi, dans sa conception classique comme dans son sens élargi, "plus souvent les dispersions de peuples liées aux malheurs politiques ou au commerce que celles entraînées par la volonté de puissance ou la pauvreté» (Schnapper, 2005: 22). Cette prééminence de la qualité de minorité économiquement dominante et/ou politiquement dominée comme critère essentiel de définition de la condition diasporique (Sheffer, 1986; Safran, 1991 ; Medam, 1993 ; Bruneau, 1995 ; Cohen, 1997) a conduit à exclure du paradigme les minorités politiquement dominantes (Sheffer, 1986), en particulier celles issues de la colonisation européenne : soit parce qu'en formant de nouvelles nations, ces dernières ont progressivement gommé tout lien avec la mère-patrie (Schnapper, 2005), comme c'est le cas des Afrikaners; soit parce qu'en étant au contraire des courroies de transmission du pouvoir de la métropole coloniale, elles ont constitué des formes éphémères du phénomène diasporique, vouées à disparaître avec la fin du projet colonial (Cohen, 1997). En résumé, les arguments avancés contre l'application du terme de diaspora aux minorités colonisatrices européennes s'appuient principalement sur la rupture de ces minorités avec leur centre d'origine et sur leur position favorable dans les relations dominants/dominés.

2 Pourtant, la diasporisation de l'ancienne minorité dominante que sont les Mauriciens blancs d'origine française (ou "Franco-Mauriciens») remet en question l'idée que dispersion colonisatrice et peuple victime seraient nécessairement antithétiques. Le groupe franco-mauricien s'inscrit en effet de façon atypique dans les relations de pouvoir, du fait de la double colonisation de l'île Maurice, d'abord par la France (1721-1810) puis par la Grande-Bretagne (1810-1967). Les Franco-Mauriciens se sont affirmés comme une minorité ethnique dominante dans le cadre de ces grands empires coloniaux, qui ont instauré dans l'île une société plurale de type " hiérocratique " avec l'esclavage (1766-1835) puis le coolie trade (1834-1907). Mais l'autorité britannique a 
favorisé l'émergence politique d'élites au sein des groupes dominés de la société plurale: groupe des "Gens de couleur» issus de la période esclavagiste; Chinois, Musulmans et Hindous arrivés dans la phase ultérieure du coolie trade. La décolonisation (1947-1968) exacerbe alors le sentiment d'un rapport de force défavorable au groupe franco-mauricien, qui voit s'effriter sa domination au profit de la majorité hindoue. La perte du monopole politique est stigmatisée par le spectre d'un "péril hindou » qui motive une émigration massive des Franco-Mauriciens, vers l'Australie et principalement vers l'Afrique du Sud.

3 L'exode de la décolonisation consacre le caractère diasporique du groupe francomauricien ${ }^{2}$. Mais nous verrons que, si celui-ci combine tous les critères propres aux diasporas, il répond toutefois à une dynamique particulière, ne pouvant s'assimiler totalement ni au modèle classique de la diaspora centrée, ni au modèle de la diaspora hybride. D'une part, le cumul d'une identité ancestrale française et d'une identité mauricienne invalide l'idée d'un modèle unitaire de diaspora fonctionnant sur le mode de la racine comme dans les diasporas classiques juive ou arménienne. D'autre part, le choix de destinations où des politiques d'immigration restrictives (Australie, Afrique du Sud) favorisent des schémas sociaux de type hiérocratique montre que la dispersion franco-mauricienne ne répond pas non plus à l'« errance » qui caractérise les diasporas hybrides.

4 La prise en compte du facteur de l'insularité comme support spécifique de la constitution de la mémoire ouvre une nouvelle voie d'analyse dans le champ paradigmatique des diasporas actuellement structuré de façon binaire entre «territoireracine " (modèle centré) et "réseau-errance » (modèle hybride) (Chivallon, 1997b). La thèse de cet article sera d'établir que la matrice insulaire, envisagée non pas comme "centre», mais comme "lieu de refondation», engendre dans le groupe francomauricien une mémoire diasporique "pollinisée », s'appuyant sur l'identité de primoarrivant.

5 La migration ancestrale venue de France constitue le «moment origine» qui fournit l'assise d'une mémoire de peuple pionnier, dans laquelle elle introduit une dialectique fondatrice entre ancrage et désir d'ailleurs (1). Le péril hindou, moteur de l'exode des Franco-Mauriciens pendant la décolonisation, réactualise ce paradoxe mémoriel dans la dispersion ${ }^{2}$. Il permet, en légitimant le ré-enracinement dans de nouveaux sols, de reproduire dans la diaspora une identité à la fois obsidionale et dominante, suivant une logique de recherche de proximité relative et de ressemblance, qu'exprime la métaphore de la pollinisation (3).

\section{La mémoire de la dispersion initiale}

6 La mémoire de la dispersion initiale est un élément cardinal de la constitution des groupes ethniques et des diasporas. Mais ethnogénèse et diasporisation sont généralement mises en tension dans l'analyse sociologique qui, dans le premier cas, considère la mémoire comme le support d'une territorialisation et dans le second, comme le moteur de la dispersion. Pourtant, il est un cadre spécifique où enracinement et dispersion ne peuvent être tenus pour mutuellement exclusifs, c'est celui de l'île : «le peuple insulaire a conservé la mémoire de son origine. Il est tout autant un peuple de voyages que de racines, un peuple de lieux qu'un peuple de routes» (Bonnemaison, 1996 : 434). 
7 L'insularité, en regroupant sur un même territoire exigu ce que les autres barrières sociologiques séparent, apparaît à la fois comme un référent commun d'identité et comme une source supplémentaire de cloisonnement, en vertu d'un «effet de condensation " propre à l'espace insulaire (Benoist, 1987). De surcroît, par le fait de vivre sur une terre irrémédiablement limitée, l'île implique nécessairement le voyage comme référent matériel d'identification, comme "contrepoint de l'enracinement et du sol» (Benoist, 1987 : 50). De ce fait, la conception insulaire de l'espace ne répond pas à une logique de centralité, mais, comme le suggère Joël Bonnemaison à propos des sociétés mélanésiennes, à un principe de «fondation»: le «lieu de fondation» est à la fois principe symbolique de l'origine dans la mémoire et point de départ d'itinéraires reliant l'île à d'autres territoires en autant de "routes d'alliance » qui ne dépendent pas tant de la proximité que de l'alliance politique préalable (Bonnemaison, 1996: 435-6). L'insularité est ainsi déterminante d'une double ambivalence : entre cloisonnement et rassemblement d'une part ; entre enracinement et voyage d'autre part.

8 L'ethnogénèse de la minorité dominante franco-mauricienne se forge dans ces contraintes liées à l'insularité, renforcées par le mode spécifique de colonisation. En effet, en l'absence de population autochtone dans l'île, la colonisation par droit de conquête entraîne la création ex nihilo d'une société nouvelle pouvant s'organiser à partir des seuls impératifs de la puissance coloniale (Benoist, 1974). Or, celle-ci s'appuie, dans l'exercice de la domination, sur l'existence d'une minorité dominante européenne ${ }^{3}$. L'institution esclavagiste fonde une stratification sociale de type « hiérocratique».

9 Les minorités dominantes tirent ainsi leur domination structurelle du rôle de courroies de transmission du pouvoir de la métropole coloniale (Benoist, 1974), la dépendance verticale de la colonie envers cette dernière marquant les limites de leurs prérogatives. Mais l'île Maurice constitue un cas atypique à cet égard, car dès les origines de la colonisation française, la minorité blanche met en place des « routes d'alliance » qui lui permettent de contourner cette dépendance verticale. Bien qu'assujettis au monopole de la Compagnie des Indes Orientales (1721-1767) par la règle de l'Exclusif qui prohibe le commerce avec les autres pays, les négociants français dans l'île contournent cet interdit en organisant un commerce régional interlope, dit commerce "d'Inde en Inde ». En même temps que ce commerce d'Inde en Inde organise le chaînage de l'île avec d'autres colonies (Inde, Madagascar, Le Cap, puis après 1803, l'Australie) en un système réticulé fondé sur la complémentarité, le système de plantation structure le contrôle territorial dans l'île au profit de la minorité blanche européenne. Cette imbrication étroite du contrôle du territoire insulaire et des routes d'alliance est le premier mécanisme de l'affirmation de la minorité dominante, et de sa relative émancipation vis-à-vis de la métropole.

10 La population blanche d'origine française ne cesse d'ailleurs de s'accroître à partir de l'abolition de l'Exclusif (1764), suivie de l'entrée en application du Code Noir (1766). Alors que les Blancs n'étaient qu'une centaine sur l'île en 1721, ils sont au nombre de 3 163 en 1767, date du premier recensement, et de 6489 en 1807 (Graphique1). Ainsi, en près de quarante ans, la population blanche double c'est sous l'effet de l'immigration, qui atteint son apogée entre 1787 et 1797, sous l'influence de la Révolution française qui provoque dans l'île un afflux d'aristocrates fuyant la Terreur. Cette part de l'immigration dans l'accroissement du groupe blanc (67 \% en 1787 et $90 \%$ en 1797) 
devient quasiment nulle après 1807 (l'île est alors sous blocus anglais), ce qui témoigne alors de l'enracinement dans le sol insulaire (Graphique 2).

11 Le mouvement migratoire de la population blanche s'inverse avec la prise de l'île par les Britanniques en 1810. Pendant la période anglaise, trois grandes vagues d'émigration affectent le groupe des colons blancs d'origine française, ce qui souligne combien la dispersion et l'enracinement, référents inhérents à l'insularité, sont également au cœur des stratégies de recomposition de la minorité dominante. Celles-ci, comme le souligne E. Kaufmann (2004), peuvent être de deux ordres: " exclusionnistes» ou "expansionnistes». Si les stratégies exclusionnistes mettent l'accent sur la préservation des spécificités ethniques du groupe, y compris par des procédés d'exclusion au sein de celui-ci, les stratégies expansionnistes cherchent à projeter leur domination vers de nouveaux territoires. Ainsi l'émigration est étroitement dépendante des mécanismes de recomposition des minorités dominantes.

Une première vague de départs (dont l'ampleur n'a jamais pu être retracée) a lieu au moment de la Capitulation. Le groupe de ceux qui restent, acceptant la prestation de serment qui fait d'eux des sujets britanniques, s'augmente par un processus d'assimilation d'éléments britanniques. Les alliances matrimoniales avec les familles britanniques fortunées contribuent, alors qu'a lieu l'essor de la monoculture sucrière à partir de 1825, à renforcer le pouvoir économique du groupe blanc d'origine française. Celui-ci parvient simultanément à évincer du foncier sucrier les Libres de couleur, l'autre groupe possédant de la société esclavagiste (Boudet, 2006b : 181).

13 Un deuxième exode, aux contours tout aussi historiquement flous que le premier, est déclenché par l'annonce de l'abolition de l'esclavage (1835) qui crée un vent de panique chez les propriétaires blancs. En sens inverse de l'émigration blanche, se produit le début de l'immigration des engagés indiens (coolie trade), mise en place par les sucriers pour remplacer les esclaves dans les champs de canne.

14 Une troisième grande vague d'émigration blanche est déclenchée par le processus industriel de centralisation de la production sucrière qui débute à Maurice en 1867. Tandis que les usines centrales et la grande plantation deviennent le quasi-monopole des grandes familles blanches, les terres de la petite plantation sont cédées aux engagés indiens, si bien que les familles blanches financièrement incapables de surmonter la concentration du capital, exclues à la fois de la grande et de la petite plantation, sont contraintes à l'émigration (Boudet, 2006a: 181-4). Le nombre de «Blancs " à Maurice, après avoir atteint son apogée en 1830, avec un total de 8592 personnes $(9 \%$ de la population totale), ne compterait plus en 1946 que 6500 personnes, soit $3 \%$ seulement de la population totale (Graphique 1).

La monoculture sucrière joue ainsi un rôle déterminant dans l'articulation des stratégies exclusionnistes et expansionnistes de l'ethnicité dominante. Elle favorise la concentration du pouvoir économique aux mains d'une plantocratie qui, en détenant $86,6 \%$ du total des terres et 92,5\% des terres cultivées en canne en 1830 (Allen, 1983 : 125), devient le «noyau dominant» de cette minorité blanche. La consolidation du monopole sucrier permet à la plantocratie d'effectuer un lobbying sur le Gouvernement colonial britannique et de se constituer à partir de 1885 en une oligarchie sucrière qui confisque le pouvoir législatif à son profit. Elle assure une pérennisation de sa position hégémonique malgré la perte des fondements structurels de la domination due à l'abolition de la barrière de couleur (1829) et de l'esclavage (1835). Le pouvoir de l'oligarchie s'exprime par sa capacité à placer sous sa coupe la gestion des finances 
publiques et par là, l'orientation de l'activité économique de l'île tout entière (Boudet, 2006a : 179).

L'émigration permet aux victimes de la concentration sucrière de développer à l'extérieur des stratégies de recomposition de la domination. Si les deux premières vagues d'émigration du début du XIX ${ }^{e}$ siècle étaient tournées essentiellement vers la France, la centralisation sucrière qui se poursuit jusqu'au $\mathrm{XX}^{\mathrm{e}}$ siècle alimente une émigration perlée vers trois terres à potentiel sucrier : l'Afrique du Sud (province du Natal), Madagascar et l'Australie (Queensland). Les flux migratoires suivent ainsi les anciennes "routes d'alliance»: route de la traite pour Madagascar, route de l'exportation des sucres mauriciens vers l'Australie et l'Afrique du Sud. Dans ces trois pays, les relations commerciales, puis la ruée vers l'or (1851 en Australie, 1886 en Afrique du Sud, 1905 à Madagascar), en attirant les aventuriers franco-mauriciens, sont le prélude à une installation durable. L'émigration vers ces pays où la barrière de couleur reste en vigueur favorise la préservation des deux «points d'érosion $»^{4}$ de la domination franco-mauricienne : la pureté raciale et le capital sucrier - le premier étant garant du second.

La migration est ainsi constitutive de l'ethnogénèse du groupe franco-mauricien en minorité dominante, non seulement comme fait social mais aussi en raison de son importance symbolique dans la construction identitaire. À cet égard, la mémoire des origines françaises du peuplement de l'île est pour les Franco-Mauriciens fondatrice d'une dualité identitaire: elle permet de "naturaliser» la communauté ethnique (Candau, 1998: 87) dans l'île, mais elle est aussi inspiratrice d'un désir d'ailleurs qui pousse à l'émigration dans les périodes de crise.

La conquête et la colonisation de l'île par la France acquiert, suite à la prise de l'île par les Britanniques, valeur de "moment origine» (Candau, 1998) ou de "moment de fondation" (Bonnemaison, 1996) pour la minorité blanche d'origine française restée dans l'île.

« Porteur de civilisation rayonnante, à l'époque où elle culminait dans le monde, l'Européen a fondé l'île » (Noël Marrier d'Unienville, 1953, L'œuvre étonnante des Mauriciens, Port-Louis, The General Printing and Stationary, 253 p., p. 59).

19 La qualité de primo-arrivant devient un argument de l'affirmation de la domination minoritaire $^{5}$ et de la lutte pour la préservation de droits spécifiques ${ }^{6}$, que ce soit dans la mobilisation contre l'abolition de l'esclavage (1831), contre l'anglicisation de l'éducation et des tribunaux $(1839,1847)$, ou encore pour la défense des prérogatives politiques :

«Les forêts ne recelaient point d'autochtones. Personne ne peut en conséquence revendiquer des droits antérieurs à ceux des Européens. [...] la fondation de la colonie fut l'oeuvre exclusive des colons français assistés de leurs esclaves d'origine africaine » (Noël Marrier d'Unienville, 1954, L'île Menacée, p. 3).

«Nos ancêtres furent les premiers occupants persévérants de l'île. Nous avons peiné pour féconder le sol, que, d'inculte qu'il était, nous avons rendu riche en moissons nourricières. Les ossements de nos aïeux ont blanchi sous la terre qu'ils avaient possédée et qu'ils nous ont léguée. De cette union indestructible entre eux et cette terre qui leur était, qui nous reste sacrée, leurs descendants tiennent leurs droits» (J. Adolphe Duclos, L'évolution nationale mauricienne, Paris, Jouve, 1924, p. 179).

L'histoire de la période française acquiert une dimension de «mytho-histoire " (Leach, 1980), c'est-à-dire une histoire qui fait fonction de mythe d'origine à valeur ontologique 
et étiologique (Eliade, 1957), en tant que modèle explicatif de la fondation de l'île et, partant, de légitimation du système social hiérocratique :

"N'oublions pas que nous sommes les gardiens d'un héritage sacré et que nos fils seraient en droit de nous demander plus tard: "Qu'en as-tu fait?" Que nous puissions alors leur répondre fièrement: "Je l'ai non seulement conservé et agrandi, mais j'ai aussi apporté le bien-être à ceux qui travaillaient pour moi". Alors nous serions certains d'avoir fait notre devoir» (Maurice A. Rey, 1944, "Employeurs et employés», in Dodo Club (Éd.), Conférences du Dodo Club de 1944, Port Louis, Nouvelle Imprimerie Coopérative, p. 59).

La mytho-histoire possède également la deuxième fonction du mythe de fondation, qui est la mise en rapport des éléments de la structure sociale (Candau, 1998). Ainsi, l'ethnonyme «Franco-Mauricien » pour désigner ceux qu'on appelait jusqu'alors les « Blancs » est forgé au début du XXe siècle dans un rapport de similitude et d'opposition à celui d'«Indo-Mauricien" qui désigne les Indiens de confessions hindoue et musulmane, devenus majoritaires de l'île (Graphique 3). La référence française favorise le glissement d'une identité raciale vers une identité ethnique (fondée sur l'ancestralité) lorsque la notion de race est ressentie comme trop hégémonique (Boudet, 2006b : 28).

22 La capacité de la mémoire des origines à mobiliser, sous la pression des circonstances, tantôt un clivage, tantôt une origine commune pour la création de nouvelles solidarités (Candau, 1998) trouve son expression dans le projet politique de la "Rétrocession », fondé en 1919. Le mouvement rétrocessionniste demande le rattachement de l'île Maurice à la France. Il est composé d'une frange du groupe blanc exclue de l'oligarchie sucrière et qui occupe des postes dans l'administration coloniale britannique ou retournée en France, et d'une élite émergente au sein du groupe « de couleur ", qui, à la suppression de la barrière de couleur, a pu accéder à l'éducation secondaire et, aux termes d'études en France, à des professions libérales de médecins, journalistes ou avocats. Les Rétrocessionnistes argumentent que le retour de l'île à la France prémunirait contre le risque d'une «menace indienne », autrement dit contre la remise en cause de la mobilité socio-économique et surtout de l'accès aux postes de l'administration par l'octroi de la citoyenneté politique aux Indiens'. C'est dans cette logique qu'ils invoquent les droits acquis par l'antériorité sur le sol de l'île ${ }^{8}$ comme critère de légitimité pour l'exercice des droits politiques.

23 Le projet rétrocessionniste se heurte à l'opposition de l'oligarchie sucrière francomauricienne, soucieuse de défendre ses marchés préférentiels en Inde et en GrandeBretagne, qu'un rattachement à la France remettrait en question. Pour les AntiRétrocessionnistes, qui s'organisent en contractant une alliance politique avec de grands propriétaires sucriers indo-mauriciens, l'argument économique prime sur l'argument culturel.

24 Le débat autour de la rétrocession polarise les identifications en mettant en jeu des alliances trans-ethniques, qui s'articulent au clivage intra-ethnique: d'une part, le noyau dominant du groupe, l'oligarchie sucrière, alliée aux grands planteurs indomauriciens contre la rétrocession; de l'autre, l'alliance en faveur de la rétrocession entre les « petits blancs", alliés à la nouvelle bourgeoisie de couleur.

Dans cette gestion des clivages intra- et inter-ethniques du groupe, ce sont les deux pôles de la mytho-histoire française qui sont mis en balance : « enracinement » contre " désir d'ailleurs ». L'échec des Rétrocessionnistes aux élections législatives de 1921, en faisant primer le premier sur le second, consacre la «communalisation» du groupe 
franco-mauricien, au sens de Max Weber, c'est-à-dire l'autonomisation d'une entité fondée sur la mémoire de la colonisation et de la migration ; l'origine française étant désormais consacrée au terme de ce processus, comme "communauté de souvenir " culturel et non plus comme communauté politique de référence. Cette communalisation du groupe par autonomisation vis-à-vis de la France dessine un projet politique fondateur d'un sentiment national, le « Mauricianisme », conceptualisé par le leader anti-rétrocessionniste Jean Adolphe Duclos :

«Nous occupons dans le monde une place à part. Nous avons une physionomie qui nous est propre. Nous possédons une faculté de rayonnement qui nous permet, bien que nous soyons une minorité, de faire pénétrer au sein des autres races qui habitent notre sol cette culture qui est nôtre. Sous les plis des deux grands drapeaux qui nous abritent, nous nous sommes assimilés des idées et des sentiments appartenant à chacune des deux races, et ce dosage d'éléments divers, harmonieusement mêlés, nous a donné un caractère spécial et a créé le mauricianisme, dont nous sommes fiers d'être les représentants. Nous sommes une entité spéciale - ni Français ni Anglais. Nous sommes Mauriciens» (J. Adolphe Duclos, 1924, L'évolution nationale mauricienne, Paris, Jouve, p. 433-4). et porte un coup définitif à l'idéal assimilationniste des proto-nationalistes francomauriciens. Face à l'octroi par l'autorité britannique de la citoyenneté politique aux nouvelles élites issues des groupes immigrants les plus récents, en particulier les anciens engagés venus de l'Inde, la peur d'un "péril hindou » réactive la dispersion comme mode opératoire d'action sociale. Mais avec la nouvelle donne engendrée par la décolonisation, le groupe franco-mauricien peut-il encore se considérer comme une minorité coloniale et pionnière?

\section{Le « péril hindou », mythomoteur de la dispersion}

Le paradigme juif, qui compose le socle des réflexions sur le phénomène diasporique, définit la diaspora archétypale comme le résultat d'une dispersion forcée et traumatique depuis un lieu d'origine - même si ce critère n'est désormais plus décisif. Le point de départ reste inscrit dans la mémoire de la diaspora comme son centre de référence, garant du maintien d'une forme d'unité malgré la dispersion (Bruneau, 2004). En revanche, dans le modèle de l'hybridité, formulé à partir de l'expérience des peuples noirs des Amériques (la «Black Atlantic » de Paul Gilroy), la traite et l'esclavage, en imposant un déracinement éradicateur par rapport au centre d'origine, ont consacré une dépossession mémorielle, compensée par une démultiplication des références suivant un mode non plus vertical, du registre de la filiation, mais transversal, animé par une pensée de l'errance (Chivallon, 1997). La dispersion franco-mauricienne se dissocie de l'un et l'autre modèles dans la mesure où la peur d'un " péril hindou » crée les conditions d'un traumatisme qui réactive une mémoire antérieure de la dispersion, mais sans que celle-ci donne lieu à une rupture mémorielle ou une errance.

La peur d'un "péril hindou ", c'est-à-dire d'une hégémonie culturelle, politique et démographique des Hindous en cas d'indépendance, motive l'exode des FrancoMauriciens en exacerbant une peur obsidionale, c'est-à-dire la peur d'être mis en 
minorité, voire assimilé ou détruit. Mais, dans la mesure où le processus de décolonisation, bien qu'émaillé par des émeutes interethniques (entre Hindous et Créoles en mai 1965, et entre Créoles et Musulmans en 1967), n'a pas donné lieu à des violences organisées envers le groupe franco-mauricien, la peur du péril hindou suffitil à affirmer l'existence d'un exode sous la contrainte et une condition de minoritaire ? Réactivation de la «menace indienne» des Rétrocessionnistes, l'idée d'un "péril hindou » est forgée entre 1953 et 1955, alors que se profile l'octroi du suffrage universel puis de l'autonomie, par Noël Marrier d'Unienville, rédacteur-en-chef du journal franco-mauricien Le Cernéen :

"Le suffrage universel ici veut dire, personne n'en doute, le suffrage hindou. Le suffrage hindou veut dire l'hégémonie hindoue. L'hégémonie hindoue signifie fatalement... l'annexion, dans un temps plus ou moins long, de l'île Maurice à l'Inde » (Noël Marrier d'Unienville, Le Cernéen du 04 juin 1953).

31 L'enjeu principal de cette période est en effet la représentation des différents groupes ethniques au sein de l'instance législative et en particulier l'introduction du principe de représentation des groupes minoritaires. Cette institutionnalisation de la représentation communautaire comme principe politique a désormais pour effet de fonder les stratégies électorales sur le principe ethnique. Elle aboutit à une polarisation de l'espace partisan entre d'une part le Parti Mauricien, identifié aux intérêts sucriers franco-mauriciens, et de l'autre le Parti Travailliste, dirigé principalement par des Hindous. L'enjeu étant dès lors de rallier les autres minorités, créole, musulmane, tamoule et chinoise, le Parti Mauricien reprend comme principe mobilisateur des minorités l'argument du " péril hindou » contre le projet d'indépendance soutenu par le Parti Travailliste.

L'argument politique de la défense des minorités marque un renversement dans les représentations $\mathrm{du}$ groupe blanc franco-mauricien, désormais présenté par ses intellectuels comme étant dans la position d'un groupe indigène ou autochtone victime d'une « colonisation à rebours» :

"Maurice a été victime, dans une certaine mesure, d'une colonisation à rebours dans laquelle les derniers venus se sont imposés aux premiers occupants, c'est-à-dire aux Français qui étaient en quelque sorte les "indigènes" de cette île déserte et aux esclaves qu'ils avaient importés après s'y être établis, aux Noirs et aux Métis. Cette colonisation à rebours consacre la domination politique des Indo-Mauriciens, fils des immigrants indiens, sur les descendants des premiers habitants et sur la population générale " (Jean-Pierre Lenoir, 1979, « Un cas limite de pluralisme : l'île Maurice », Cultures 6 (1), pp. 65-85 : 82-3).

Cependant, l'alliance politique avec les minorités asiatiques (tamoule, chinoise et musulmane) considérées comme «dernières venues" sur le sol mauricien, rend désormais impossible la politisation de l'argument autochtoniste (ou par extension, de la notion de primo-arrivant) comme principe de légitimité politique. En effet, le recours à un tel argument d'antériorité aurait impliqué, en même temps que l'énonciation d'une supériorité du groupe blanc, la disqualification de ces minorités de la citoyenneté nationale (Ceuppens et Geschiere, 2005) alors qu'il est au contraire devenu nécessaire de les rallier. L'alliance entre minorités justifie alors l'élision du caractère dominant de la minorité blanche en politique.

Dans les faits, le groupe blanc correspond difficilement à la définition de minorité, qui implique une difficulté d'accès aux postes de contrôle des activités économiques et politiques (Schermerhorn, 1968), excepté si l'on s'en tient à la définition d'origine de Louis Wirth (1945) selon laquelle est minoritaire un groupe qui se considère comme 
victime d'un traitement discriminatoire de la part du reste de la société. Malgré l'inexistence institutionnelle du groupe blanc - absent des statistiques officielles qui l'englobent avec les Créoles dans une catégorie «Population Générale»- et sa faiblesse numérique, la participation de son élite au grand accord de la consociation (Boudet, 2003) continue de lui assurer une pérennisation de sa représentation politique et de sa domination économique (Boudet, 2006b). C'est donc la production discursive autour d'un péril hindou qui confère à l'ancienne minorité dominante cette dimension victimaire de la qualité de minoritaire.

La peur obsidionale d'un péril hindou entretenue par le politique a prise sur les mentalités franco-mauriciennes en raison de la méconnaissance réciproque qui prévaut au sein de la société plurale, ethniquement et économiquement cloisonnée ${ }^{9}$. Le péril hindou fragilise d'autant plus le groupe dans son imaginaire que sa mobilité sociale au sein du secteur sucrier est verrouillée par le système de protections entretenu par les grandes familles (Boudet, 2004 : 12). Entre 1945 et 1968, plus de 2814 migrants officiels appartenant essentiellement au groupe blanc quittent Maurice pour l'Afrique du Sud. Les Franco-Mauriciens, qui étaient au nombre de 6500 dans l'île en 1945, ne seraient plus en 1969 , quand l'exode a pris fin, que $3700^{10}$. Leur part dans la population totale de l'île passerait ainsi de $3 \%$ à seulement 0,5\% (Boudet, 2006a : 38). Comparativement, ils sont estimés en 1981 à plus de 12000 dans la seule province du KwaZulu-Natal, en incluant la seconde génération (Bullier, 1981b). L'exode de la décolonisation, se greffant sur l'émigration perlée déclenchée par la centralisation sucrière du siècle précédent, consacre ainsi un critère essentiel de la condition diasporique: l'accentuation du caractère ultra-minoritaire du groupe par la perte de plus d'un tiers de ses effectifs et l'inversion du rapport numérique de sa représentation entre l'île et l'extérieur.

La période de la décolonisation renouvelle aussi les termes de la dispersion. Jusqu'alors, les Franco-Mauriciens étaient pratiquement les seuls à émigrer (Dinan, $1985: 11$ ) et leur migration s'effectuait principalement vers deux ou trois pays : l'Afrique du Sud, l'Australie et dans une moindre mesure Madagascar. Dans les années soixante, l'Afrique du Sud est devenue leur principale destination, mais l'exode franco-mauricien s'inscrit désormais dans une dispersion qui touche toutes les minorités: Chinois, Tamouls, Musulmans (Vuddamalay, 1993 : 195) et qui s'effectue vers un éventail de destinations d'Europe, d'Amérique du Nord, du Moyen-Orient ${ }^{11}$. La dispersion revêt d'après $\mathrm{V}$. Vuddamalay un caractère de spécialisation ethnique liée à la spécialisation professionnelle des différents groupes (Vuddamalay, 1993: 195-2000), en fonction de laquelle l'Afrique du Sud, si elle ne compte plus après 1965 que pour $3,5 \%$ de l'émigration totale officielle, reste identifiée comme le monopole des FrancoMauriciens, qui y sont déterminants dans le développement de l'industrie sucrière.

Le moteur de la dispersion franco-mauricienne des années de la décolonisation est donc moins la condition de minoritaire que son instrumentalisation politique. L'exode marque les limites de cette instrumentalisation et apparaît comme l'effet pervers du discours électoral sur le péril hindou: au lieu de mobiliser autour d'une communauté de danger, ce dernier suscite la désolidarisation. Or, la mobilisation sociale, pour être effective, impose que soient activés à la fois le clivage culturel (nécessaire à la relation d'altérité) et la notion de communauté d'intérêt interne au groupe (Nettl, 1967; Oberschall, 1973). L'argument du péril hindou facilite la réalisation de la première condition alors que la seconde reste manquante en raison de la concentration du pouvoir aux mains de l'oligarchie sucrière. L'argument électoral d'un péril hindou a 
ainsi conduit à l'émigration parce qu'au lieu de polariser les identifications, il a favorisé l'éclatement des agrégats de l'identité franco-mauricienne.

Pour autant, la dispersion donne-t-elle lieu à une mémoire itinérante qui construirait des modes alternatifs de relation au territoire et à l'État-nation, comme le suggère le modèle de l'hybridité ? Ou implique-t-elle, comme l'entend le paradigme classique, la rupture vis-à-vis du politique territorialisé comme condition de la conscience diasporique ? L'État-nation constitue le point de référence des diasporas, soit qu'il leur fournisse, comme dans le modèle centré, le moyen de maintenir une forme d'unité à travers la dispersion; soit que la conscience diasporique se construise, comme dans le modèle hybride, en tension avec l'affiliation aux modes de pouvoir de l'État-nation. La diaspora s'affirmerait dans ce deuxième cas de figure comme une «culture nomade » (Gilroy, 1994), détachée de son ancrage territorial et existant en dehors de, voire même en opposition aux codes et formes politiques de la citoyenneté moderne.

Ce rapport de la diaspora au politique étatique a conduit les auteurs à considérer le modèle diasporique comme s'opposant au principe d'autochtonie (Bruneau, 2004; Centlivres, 2005). L'État-nation se fonde sur un principe d'autochtonie, élaborant son identité en relation avec un territoire qui lui préexiste et affirmant la permanence de cette relation; tandis que dans la diaspora au contraire, l'identité préexiste généralement au lieu, et le groupe en diaspora va donc chercher à reproduire dans le lieu d'accueil cette identité (Bruneau, 2004). Or, dans le cas de la dispersion francomauricienne, le modèle diasporique ne s'établit pas sur cette césure inhérente aux deux grands modèles paradigmatiques.

La diaspora franco-mauricienne n'apparaît pas comme " une rupture avec l'affirmation du primat de l'autochtonie et de l'enracinement" (Centlivres, 2005). L'identité de primoarrivant, si elle n'a pas servi de principe de mobilisation politique, légitime l'émigration comme une forme protestataire de réponse à la décolonisation. Le péril hindou qui confère un caractère obsidional à l'identité de primo-arrivant, " colonisé à rebours ", à son tour renforce l'idée d'une occupation du sol insulaire faite sans violence et sans subjugation : «[...] nous sommes arrivés à Maurice en premier et personne ne peut remettre ça en question. Nous ne sommes pas des colonialistes, nous ne sommes pas des impérialistes. Je dis ça à mes enfants : n'oubliez jamais ça ", souligne un Franco-Mauricien émigré à Durban en 1970 (cité dans Boudet, 2004 : 434). En justifiant et en entretenant une conscience de minoritaire victime, le péril hindou fonctionne comme un mythomoteur, c'est-à-dire comme un mythe à vocation étiologique susceptible de favoriser et de motiver l'affirmation d'une conscience identitaire (Armstrong, 1982). À la différence près que ce mythomoteur, au lieu de structurer l'appartenance territoriale comme dans le modèle d'Armstrong, organise sa désolidarisation. Celle-ci s'effectue vis-à-vis du nouveau régime politique mais non du sol insulaire, auquel la qualité de primo-arrivant, de fondateur d'une société ex nihilo, justifie le rattachement immémorial et indéfectible.

41 Le péril hindou dans sa justification d'une conscience de primo-arrivant suscite un mode particulier de dispersion qui fonctionne sur le mode du tropisme. La province sud-africaine du Natal en particulier réunit les conditions d'un "tropisme sudafricain » (Bullier, 1981b : 266) résultant d'un ensemble d'affinités, à la fois politiques et économiques, et qui permettent aux migrants de partir vers un espace ressenti comme proche, accessible, car favorable à la fois à la préservation de la domination ethnicoraciale et à la réussite sucrière. 
42 Le sentiment d'anomie sociale et d'insécurité lié aux tensions du processus constitutionnel à Maurice contraste dans l'esprit des candidats à l'émigration avec la perception d'une Afrique du Sud où règne un ordre social favorable aux Blancs et sécurisé. Le régime d'apartheid qui prévaut alors en Afrique du Sud, en instaurant la barrière de couleur dans l'accès à l'espace résidentiel, au système éducatif et au marché du travail, contribue à canaliser l'intégration des immigrants franco-mauriciens au sein du groupe dominant de la société sud-africaine (Boudet, 2006a : 40).

Le sentiment de proximité est de surcroît alimenté par le rôle pionnier qu'ont joué dans l'édification de l'industrie sucrière au Natal depuis 1848 les Franco-Mauriciens, présents dès la mise en valeur de terres de la colonie par les Britanniques (Boudet, 2004, 2006a). De sorte que l'identité franco-mauricienne n'a pas préexisté au lieu d'installation, elle s'est construite simultanément à celui-ci, l'imaginaire du groupe dans l'île s'alimentant aussi de l'histoire coloniale sud-africaine :

"Pour le Sud-Afrique, j'ai commencé à rêver de ses territoires quand, petit garçon de $12 \mathrm{ou}$ 13 ans, les coups de canon de la guerre anglo-boere parvenaient jusqu'à mon île, électrisant grands et petits. Des mots inconnus devenaient tout à coup familiers. On ceignait la cartouchière des burghers pour accompagner les commandos dans le veld, les kopjes s'illuminaient de la lueur des fusils, on citait des noms fameux de batailles [...] Je réponds à l'appel ancien" (Clément Charoux, 1939, Vacances sud-africaines, Port-Louis (Maurice), Editions de la Société des Ecrivains Mauriciens, The General Printing And Stationary, p. 46).

44 Cette simultanéité du processus migratoire et identitaire contribue ainsi à ancrer dans la mémoire du groupe une identité de pionnier intégrant le Natal comme lieu de refondation:

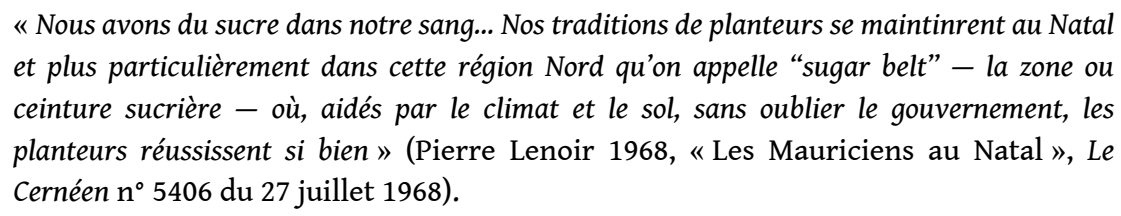

45 Fonctionnant comme un mythe de la terre promise, le tropisme natalien apparait comme le pendant positif du péril hindou. Il fait fonction de second mythomoteur dans la mesure où le nouvel exode (de Maurice vers l'Afrique du Sud) reproduit à l'identique, mais sur des bases nouvelles, la saga initiale de l'exode initial et du premier réenracinement. Ce double mythomoteur permet que se constitue la double tension spatio-temporelle qui conditionne l'existence d'une diaspora: la tension vers un ailleurs originel (sentiment d'exil à partir d'un territoire d'origine) et celle vers un futur idéalisé sur une "Terre promise", fait d'attentes liées à ce passé fondateur (Bruneau, $2004: 11$ ).

46 Toutefois, en motivant une émigration qui ne s'effectue pas au hasard sur le mode d'une "errance ", mais en choisissant une destination tenue pour sûre, ce mode de dispersion renvoie ainsi dos à dos le paradigme de la racine et celui du rhizome dans le développement de la diaspora franco-mauricienne.

\section{Une diaspora « pollinisée »}

Les modèles archétypaux voient dans le territoire d'origine la référence commune garante du maintien d'une forme d'unité au sein de la diaspora, dont la continuité serait ainsi assignable à une source, une origine (suivant la métaphore de la racine). Le 
modèle de l'hybridité, qui décrit les diasporas noires issues de la traite et de l'esclavage, suggère au contraire une dispersion caractérisée par une logique réticulaire, suivant la métaphore du rhizome, et entrainant une constante mixité de références et une mobilité à l'œuvre jusque dans les construits identitaires (Chivallon, 1997a).

La fin en 1994 du régime d'apartheid en Afrique du Sud réactive la problématique dispersion/enracinement en faisant écho au traumatisme du péril hindou. Les anciens immigrants franco-mauriciens des années 1960 ont l'impression de revivre les événements de la décolonisation à Maurice, les mêmes peurs : «face à ce qui se passe ici, je ressens exactement ce qui s'est passé à Maurice dans les années 1960, ce sont exactement les conversations qui se tenaient dans les salons à Maurice, sur la peur, sur les problèmes pour l'éducation des enfants, etc., je retraverse ce que j'ai traversé à cette époque " témoigne une Franco-Mauricienne installée à Durban depuis 1965 (cité dans Boudet, 2004 : 373). La transition démocratique en Afrique du Sud est alors perçue comme la chute du dernier bastion des valeurs de la colonisation. Les départs liés au changement politique ont lieu parmi les immigrants les plus récents, ceux dont les familles sont arrivées dans le pays avec l'exode des années soixante, tandis que la migration de souche plus ancienne est moins réversible, moins perméable au changement politique (Boudet, $2004: 373$ ).

L'émigration franco-mauricienne post-apartheid s'inscrit dans le brain drain qui affecte le groupe blanc sud-africain (Meyer, 1999: 15-7) dont la dispersion s'effectue principalement vers l'Australie, l'Europe et le continent nord-américain. Elle donne également lieu à une rémigration. Mais les retours vers Maurice alternent aussi avec de nouvelles vagues de départ, si bien que ces marées migratoires entre Maurice et l'Afrique du Sud prennent la forme d'un balancier, réagissant aussi à l'évolution politique mauricienne ${ }^{12}$.

50 Ainsi, la diaspora franco-mauricienne ne rejette pas l'allégeance au territoire ou au politique au profit d'une mémoire itinérante. C'est plutôt une forme alternative d'appartenance nationale et de citoyenneté qui se joue, dans laquelle la qualité de primo-arrivant constitue une "forme souple et malléable d'ethnicité" (Geschiere et Nyamnjoh, 2000), permettant aussi bien la perpétuation de l'identité dans le pays d'accueil, que la re-dispersion en cas de nécessité.

51 Le lien diasporique est maintenu au niveau transnational, entre Maurice, l'Afrique du Sud et les autres pays de rayonnement (principalement l'Australie, la Nouvelle-Zélande, la France, l'Angleterre, les États-Unis), par la prééminence du lien familial. Le caractère trans-national des familles franco-mauriciennes est souligné par la complexité des situations de nationalité chez leurs membres. Outre les cas de double nationalité chez les Franco-Mauriciens (française/mauricienne, mauricienne/sud-africaine, française/ sud-africaine, britannique/sud-africaine), on note au sein même des familles la coexistence de nationalités diverses, pouvant aller jusqu'à cinq ou six nationalités et pays d'installation différents au sein d'une même génération (Boudet, $2004: 375)$. Cette dissociation entre la nationalité, en tant qu'appartenance à une communauté culturelle, et la citoyenneté, en tant qu'allégeance au lieu contractuel à l'État-nation, témoigne de cette forme supranationale de parenté que l'on retrouve aussi dans la diaspora hybride (Gilroy, $1994: 210$ ).

Cependant, contrairement au modèle hybride, il ne s'agit pas d'une démultiplication de références identitaires à l'infini. L'identité de la diaspora franco-mauricienne repose au contraire sur une sélection définie de pôles fondateurs qui permettent de conserver la double référence au territoire d'origine (Maurice) et au pays ancestral (la France), c'est- 
à-dire à la fois la mémoire de la dispersion ancestrale et celle de l'enracinement : la francophonie (rôle de vecteur linguistique et de cadre culturel), la race (marqueur de la domination), le caractère de primo-arrivant (gage de reproductibilité du caractère pionnier), la religion catholique (cadre de valeurs) et le sucre (garant de la réussite).

Ces pôles sont beaucoup plus que de simples marqueurs identitaires ou repères garants d'une stabilité : dans la mesure où ils constituent les marqueurs de la permanence de l'origine dont la mémoire diasporique a besoin pour construire son identité de groupe (Candau, 1998: 90), ils apparaissent comme des modes d'emploi efficaces et réactualisables de la situation diasporique. Ils permettent qu'au fur et à mesure de la migration, se reconstruisent en mode perlé les modalités de la refondation. Le registre des pôles disponibles permet à chaque segment de la diaspora de s'identifier à un pôle ou à l'autre, voire de les combiner, sans pour autant céder à une logique de démultiplication. Selon les contextes à l'aune desquels est réinterprétée l'identité, l'un ou l'autre pôle pourra rester dormant ou être réactivé. Ainsi, la référence au caractère de primo-arrivant dans l'île permet que perdure l'identité à la seconde génération en Afrique du Sud, malgré la disparition éventuelle, selon les trajectoires individuelles, de l'un ou l'autre marqueur ethnique. Même quand la langue française s'est perdue, la mémoire de la filiation par rapport à l'ancêtre bâtisseur, le premier de la lignée arrivé dans l'île, permet la projection symbolique de qualités qui fondent la différence (favorable) du groupe sur les autres groupes de la société sud-africaine, et sert des objectifs de négociation d'avantages économiques et de reconnaissance sociale (Boudet, 2004 : 441).

54 Ces pôles ou ancrages identitaires, éprouvés à l'aune des schémas culturels de la société d'accueil, s'alimentent à la mytho-histoire du groupe tout autant qu'ils en constituent un prolongement dans la diaspora, renouvelant sa vocation ontologique et étiologique. La réinterprétation de l'histoire des origines de la dispersion donne un sens aux changements historiques vécus en Afrique du Sud, en établissant une continuité entre passé migratoire et présent. En 1985, le prêtre franco-mauricien Gérard de Fleuriot et aumônier de ses compatriotes dans la paroisse de Saint-Louis à Durban, rattache la décolonisation qui a poussé les Franco-Mauriciens à émigrer à une longue chaîne historique de périodes charnières :

« Nos ancêtres connurent des moments similaires, où ils eurent à se détacher d'un passé qui leur était familier pour s'adapter à des réalités nouvelles. Ils connurent l'expatriation, l'abolition de l'esclavage et bien d'autres changements de régimes qui n'étaient pas sans affecter leur mode de vie et de pensée [...] ( (Gérard de Fleuriot, bulletin Saint Louis Parish, de mars 1985).

L'histoire des origines fournit aussi la possibilité d'y puiser les modèles de comportement. Dans une allocution prononcée en 1975, le Franco-Mauricien Alex Bax, alors consul honoraire de France à Durban, revisite la référence à l'esclavage, en la réintroduisant dans l'histoire du groupe comme trait primordial le rattachant à l'histoire de l'île. Il établit un parallèle entre la destinée de victime de l'esclave, et la condition des Franco-Mauriciens en Afrique du Sud face à la menace de disparition pesant sur la langue française en milieu anglophone :

"[...] juché sur ce podium, conscient de ma nudité culturelle aux yeux d'auditeurs tellement mieux qualifiés, je ressens avec acuité le sentiment de l'esclave sous le marteau du commissaire-priseur, aux origines de la colonisation mauricienne. Les mains liées, il n'a que sa chair à offrir, et, conscient de la médiocrité de cette offre, il promène sur l'assistance distinguée un regard incrédule, se disant: "Mais que diable suis-je venu faire dans cette galère?" [...] Ce soir, seul et nu sous la coupole de la science, un rayon plus glorieux encore 
me libère de ma lourdeur infime : je suis appelé à contribuer à l'ouvre magistrale de sauver la culture française en grave péril chez les milliers de compatriotes du Natal! Transformé par cet idéal exaltant, ce n'est plus l'esclave qui vous offre sa chair, c'est le pélican qui vous offre son cour» (Alex Bax, "La langue française et les Mauriciens du Natal», Comptes Rendus trimestriels de l'Académie des Sciences, 35 (4), p. 605). 
d'une sécurité dans le monde des affaires, dans le contexte sud-africain post-apartheid où la corruption signale un échec de l'État (Boudet, 2004 : 444).

61 La notion de relative proximité et de ressemblance, centrale dans la dynamique de la diaspora pollinisée ${ }^{14}$, permet au groupe de «maintenir ce qui [peut] demeurer stable et s'adapter aux changements qu'il ne [peut] contrôler » (Beaudoux-Kovats et Benoist, 1972 : 111) y compris à travers la dispersion, qui constitue la stratégie ultime pour conserver ses caractères tout en allant chercher ailleurs des conditions favorables à sa survie.

\section{Conclusion}

$62 \mathrm{Ni}$ « identité-racine » fondée sur la reproduction d'une unicité mythique, ni « identitérelation ", fondée sur l'errance et la démultiplication des références, l'identité de la diaspora franco-mauricienne opère sur le mode de la pollinisation croisée. Contrairement au modèle du rhizome qui se caractérise par l'absence de pôles, ne pouvant donc produire que des formes d'identité variables étrangères à toute fixation (Chivallon, 2004), et au modèle de la racine, qui se caractérise par un noyau dur identitaire, le modèle pollinisé se caractérise par un ensemble de pôles en interaction, qui établissent des références culturelles perpétuellement réactualisables et combinables, permettant à la diaspora de survivre aux décadences successives de la décolonisation et de lui conférer une permanence temporelle et spatiale à travers de nouveaux enracinements.

La qualité de primo-arrivant est déterminante de la relation atypique que la diaspora pollinisée franco-mauricienne entretient avec l'État-nation. Alors que le modèle hybride de la diaspora rejette toute référence à la nation, celui de la pollinisation pose l'enracinement initial comme argument de légitimité d'une appartenance primant sur tout régime politique considéré comme contingent, et vis-à-vis duquel on peut donc se désolidariser sans que cela implique une rupture avec la matrice mauricienne.

Le mythomoteur du " péril hindou » favorise la réactualisation du caractère de primoarrivant et la conservation d'une identité de peuple-victime minoritaire propre à maintenir la conscience et le lien diasporique. En sollicitant comme contrepoint positif un tropisme natalien, il fonctionne selon la dialectique de la refondation. La matrice insulaire mauricienne n'est pas un centre à partir duquel se créent des périphéries, mais point nodal de l'appartenance, où s'est réalisé le succès du groupe et qui en garantit donc la reproductibilité. Lieu de fondation qui appelle d'autres lieux de refondation, elle favorise une forme de dispersion qui revêt un certain degré d'imprédictibilité, mais qui n'est pas erratique comme peut l'être le modèle rhizomique. La métaphore de la pollinisation croisée permet de rendre compte du fait que la condition essentielle de l'interaction pour le groupe franco-mauricien est l'idée de proximité relative et de ressemblance.

65 Si la qualité de primo-arrivant n'est pas inscrite comme principe de légitimité politique, elle reste, du fait du recours à l'argument du « péril hindou », inscrite dans la mémoire et dans l'identité du groupe franco-mauricien y compris en diaspora, comme "forme primale d'appartenance" permettant d'offrir "une sécurité élémentaire liée à l'ancrage des racines " (Geschiere, 2006: 2). Cette forme de primordialisme permet au groupe et à sa diaspora de s'insérer dans les frontières constamment changeantes créées par la dispersion et par les changements de nature de l'État. Cette sécurité de 
l'ancrage conservée dans la mémoire malgré la dispersion devient paradoxalement la justification même de cette dispersion.

\section{BIBLIOGRAPHIE}

ALLEN Richard B. (1983) Creoles, Indian Immigrants and the Restructuring of Society and Economy in Mauritius, 1767-1885, Ph. D. Thesis, Histoire, University of Illinois, 293 p.

ARMSTRONG John A. (1982) Nations before Nationalism, Chapel Hill, University of North Carolina Press, $411 \mathrm{p}$.

BEAUDOUX-KOVATS Edith et BENOIST Jean (1972) Les Blancs créoles de la Martinique, in Jean Benoist Éd., L'archipel inachevé : culture et société aux Antilles françaises, Montréal, Presses de l'Université de Montréal.

BENOIST Jean (1987) L'insularité comme dimension du fait social, Iles tropicales, Insularité, Insularismes, Collection « îles et archipels » (8), Talence, CRET, pp. 38-51.

BENOIST Jean (1974) Perspectives pour une connaissance des sociétés contemporaines des Mascareignes et des Seychelles, Annuaire des Pays de l'Océan Indien,(1), pp. 223-233.

BONNEMAISON Joël (1996) Les fondements géographiques d'une identité. L'archipel du Vanuatu, (1) Gens de pirogue et gens de la terre, Paris, ORSTOM, $460 \mathrm{p}$.

BOUDET Catherine (2003) L'émergence de la démocratie consociative à Maurice (1948-1968), Annuaire des Pays de l'Océan Indien, (17), pp. 325-336.

BOUDET Catherine (2004) Les Franco-Mauriciens entre Maurice et l'Afrique du Sud: identité, stratégies migratoires et processus de recommunaurisation, Thèse de Doctorat, Science Politique, Institut d'Etudes Politiques, Bordeaux, $642 \mathrm{p}$.

BOUDET Catherine (2006a) Une minorité ethnique dominante face à la décolonisation :

l'émigration franco-mauricienne en Afrique du Sud (1947-1968), Journal of Mauritian Studies New Series 3 (1), pp. 26-49.

BOUDET Catherine (2006b) Pouvoirs et technologies en situation coloniale : les FrancoMauriciens et le monopole des technologies sucrières à Maurice et au Natal (1825-1968), Revue Historique de l'Océan Indien, (2), pp. 178-193.

BRUNEAU Michel (1995) Espaces et territoires de diaspora, in Michel Bruneau Éd., Diasporas, Montpellier, GIP-Reclus, pp. 5-23.

BRUNEAU Michel (2004), Diasporas et espaces transnationaux, Paris, Anthropos, 249 p.

BULLIER Antoine (1981a) Le parler franco-mauricien au Natal : une enclave francophone en Afrique du Sud: éléments d'une phonologie, Paris, L'Harmattan, $181 \mathrm{p}$.

BULLIER Antoine (1981b) La communauté franco-mauricienne en Afrique du Sud, Annuaire des Pays de l'Océan Indien, (8), pp. 265-273.

BURKEY Richard M. 1978, Ethnic and Racial Groups. The dynamics of dominance, Menlo Park, NJ, Cummings. 
CANDAU Joël (1998) Mémoire et identité, Paris, PUF, 225 p.

CENTLIVRES Pierre (2005) Portée et limites de la notion de diaspora, CEMOTI (30) dossier « Les diasporas ».

CEUPPENS Bambi et GESCHIERE Peter (2005) Autochtony : Local or Global ? New modes of struggle over Citizenship and Belonging in Africa and Europe, Annual Review of Anthropology (34), pp. 385-407.

CHIVALLON Christine (1997a) De quelques préconstruits de la notion de diaspora à partir de l'exemple antillais, Revue Européenne des Migrations Internationales, (13) 1, pp. 149-160.

CHIVALLON Christine (1997b) Du territoire au réseau : comment penser l'identité antillaise ?, Cahiers d'Etudes Africaines, 37-4 (148), pp. 767-794.

COHEN Robin (1997) Global diasporas, Cornwall, UK, UCL Press, 227 p.

DINAN Monique (1985) Une île éclatée : analyse de l'émigration mauricienne - 1960-1982, Port-Louis (Maurice), Best Graphics, 239 p.

DOANE Ashley W. Jr (1997) Dominant Group Ethnic Identity in the United States : the Role of Hidden Ethnicity in Intergroup Relations, Sociological Quarterly, 38 (3), pp. 375-397.

ELIADE Mircea (1957) Mythes, rêves et mystères, Paris, Gallimard, 279 p.

ERIKSEN Thomas Hylland (1993) Ethnicity and Nationalism : Anthropoligical Perspectives, Londres, Boulder, Pluto press, $179 \mathrm{p}$.

GEORGE Pierre (1984) Géopolitique des minorités, Paris, PUF, 127 p.

GESCHIERE Peter (2005) Autochtony and Citizenship : New Modes in the Struggle over Belonging and Exclusion in Africa, Forum for Development Studies (2), pp. 371-384.

GILROY Paul (1994) Diaspora, Paragraph, 17 (1), pp. 207-212.

KAUFMANN Eric p. (2004) Introduction, in Eric p. Kaufmann Éd., Rethinking ethnicity. Majority groups and dominant minorities, Londres et New York, Routledge, $254 \mathrm{p}$.

LEACH Edmund R. (1980) L'unité de l'homme et autres essais, Paris, Gallimard, 389 p.

MEDAM Alain (1993) Diaspora/Diasporas. Archétype et typologie, Revue Européenne des Migrations Internationales, 9 (1), pp. 59-65.

MEYER Jean-Baptiste (1999) Expatriation des compétences africaines : « l'option diaspora » de l'Afrique du Sud, Afrique Contemporaine, (190) pp. 3-19.

NETTL John Peter (1967) Political Mobilization : A Sociological Analysis of Methods and Concepts, Londres, Faber, $442 \mathrm{p}$.

OBERSCHALL Anthony R. (1973) Social Conflict and Social Movements, Englewood Cliffs, PrenticeHall, $371 \mathrm{p}$.

PREVELAKIS Georges (1996) Les réseaux de diaspora - Introduction, inG. Prevelakis (Éd.), Les réseaux de diasporas, Paris, L'Harmattan, pp. 29-34.

SAFRAN William (1991) Diasporas in Modern Societies : Myths of Homeland and Return, Diasporas, (1), pp. 83-99.

SCHERMERHORN Richard A. (1968) Comparative Ethnic Relations, A Framework for Theory and Research, New York, Random House, 325 p. 
SCHNAPPER Dominique (2001) De l'État-nation au monde transnational. Du sens et de l'utilité du concept de diaspora, Revue Européenne des Migrations Internationales, (17) 2, pp. 9-36.

SCHNAPPER Dominique (2005) De l'État-nation au monde transnational. Du sens et de l'utilité du concept de diaspora, in Lisa Anteby-Yemini, William Berthomière et Gabriel Sheffer (Eds.), Les diasporas 2000 ans d'histoire, Rennes, PUR, pp. 21-50.

SHEFFER Gabriel (Éd.) (1986) Modern diasporas in international politics, New York, Saint Martin's press, $349 \mathrm{p}$.

SIMMONS Adele S. (1982) Modern Mauritius. The politics of decolonization, Bloomington, Indiana University Press, $242 \mathrm{p}$.

WIRTH Louis (1945) The problems of minority groups, in R. Linton (Éd.), The Science of Man in the World Crisis, New York, Columbia University Press, p. 347.

\section{ANNEXES}

Graphique 1 : Évolution de la population blanche de l'île Maurice (1721-1969)

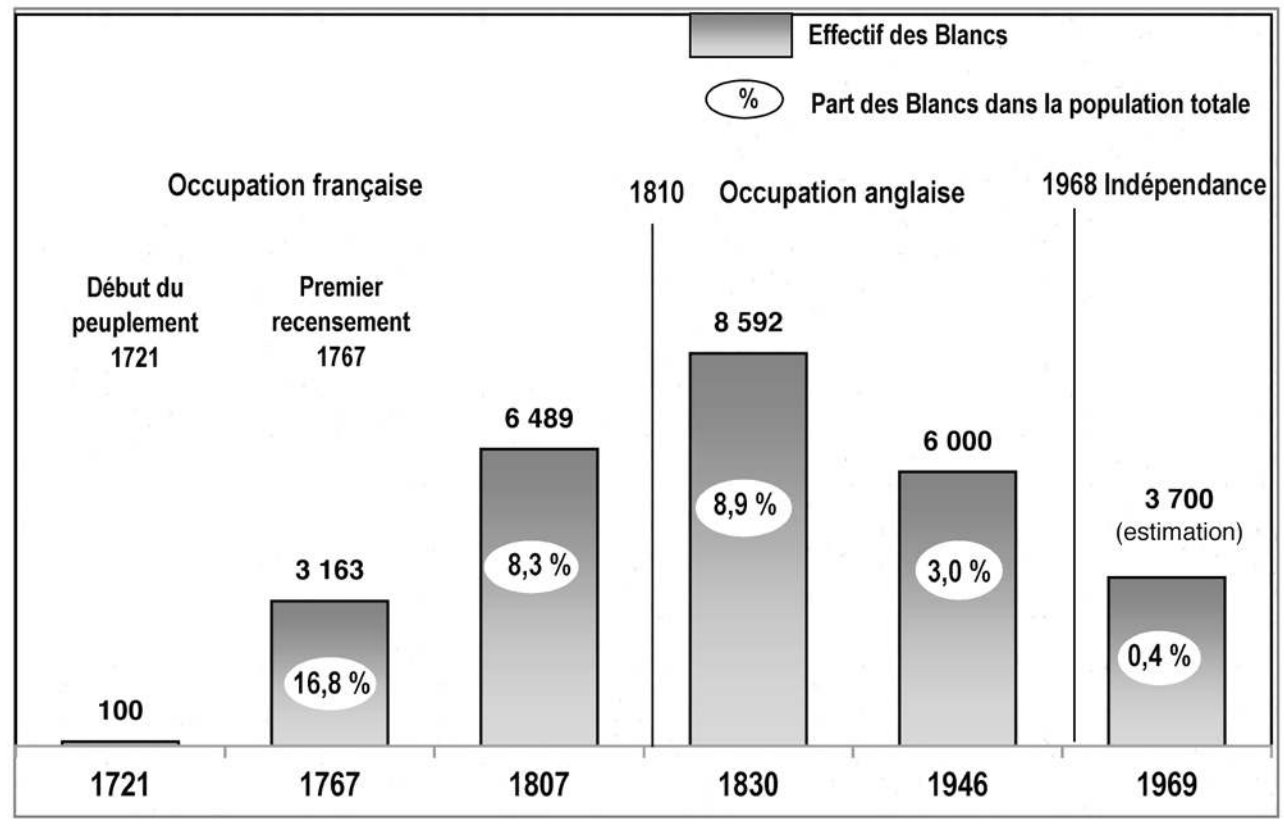

Réalisation : I'auteur, d'après : Recensements / pour l'année 1946 : Le Cernéen-Le Mauricien-Advance n 1626 du 20 octobre 1947. 
Graphique 2 : Accroissement de la population blanche de l'île Maurice (1777-1827)

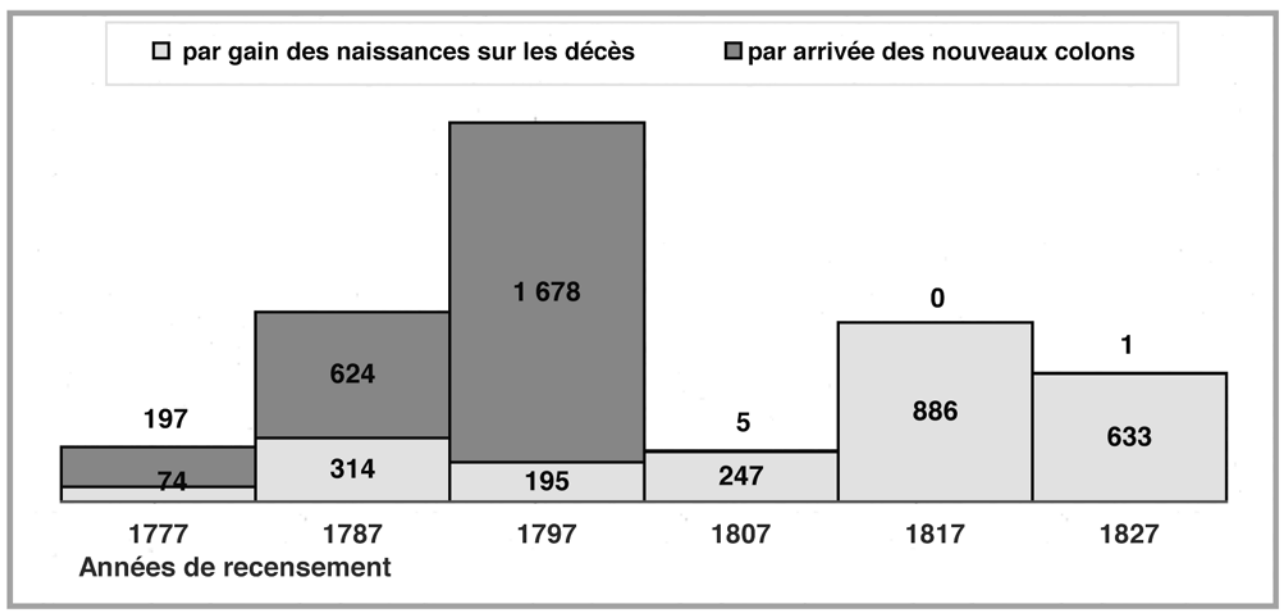

Réalisation : l'auteur, d'après Baron d'Unienville 1826, Statistiques de l'île Maurice et de ses dépendances, Vol. 3, Tab. 6511.

Graphique 3 : Catégorisation de la population selon les recensements (1767-1972) (part de chaque communauté recensée)

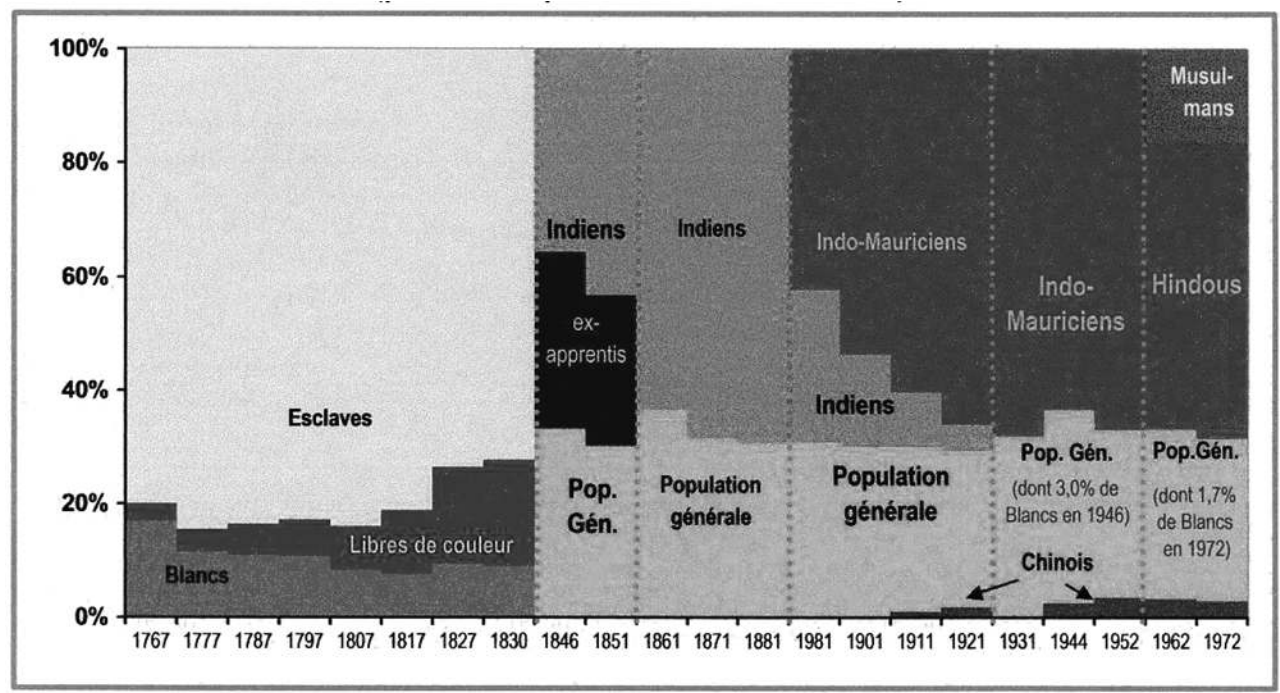

Réalisation : I'auteur, d'après Annal Digest of Statistics, 1998, CSO, Ministère de la planification / ALLEN R. B. 1993, Creoles, Indian Immigrants and the Restructuring of Society and Economy in Mauritius, 1767-1885 (Ph. D. Thesis : Histoire : University of Illinois).

\section{NOTES}

1. C'est-à-dire qui s'organise de façon coercitive en une pyramide hiérarchisant des groupes économiquement complémentaires mais considérés comme socialement, culturellement, voire racialement inégaux, sous la domination de la minorité européenne (Schermerhorn, 1968).

2. Avec l'exode de la décolonisation, le groupe franco-mauricien remplit les principaux critères constitutifs des diasporas archétypales (Boudet, 2004) : la dispersion traumatique à partir d'un centre historique; la préservation de la mémoire de ce territoire d'origine, doté d'une valeur 
quasi-mythique; la notion d'exil sous la contrainte et l'exode massif qui ont pour résultat la dispersion du groupe vers plusieurs pays d'accueil et le fait que ses membres soient numériquement plus nombreux que ceux restés sur le territoire national (George, 1984 ; Bruneau, 1995) ; la durée de la dispersion dans le temps, même si les circonstances qui ont été à son origine étaient conjoncturelles (Médam,1993); le caractère réticulaire de la diaspora qui assure sa préservation culturelle et économique (Prévélakis, 1996); la rémigration (Domenach et Picouet, 1992) et enfin, la participation à la vie socio-économique du pays d'accueil (Schnapper, 2001).

3. Le concept de minorité dominante fait référence à l'exercice par un groupe ethnique minoritaire d'une domination politique et/ou culturelle à l'intérieur d'un État (Kaufmann, 2004 : 1). La minorité dominante se définit par sa capacité à instaurer un schéma de domination qui aboutit à une distribution inégale des ressources politiques, économiques et institutionnelles et au façonnement des modes de pensée en vigueur dans la société de manière à faire accepter cet état de fait (Doane, 1997 : 376).

4. Nous empruntons cette notion de «points d'érosion » de la domination ethnique à E. BeaudouxKovats et J. Benoist à propos des Békés de Martinique, autre minorité blanche dominante fortement endogamique et fondée sur le pouvoir sucrier (Beaudoux-Kovats et Benoist, 1972 : 131). Toutefois, l'éruption de la Montagne Pelée en 1905, en décimant le groupe béké, a favorisé la mobilité ascendante de ses survivants, si bien que celui-ci, contrairement aux FrancoMauriciens, n'a jamais ressenti la nécessité d'émigrer pour recomposer les fondements de sa domination.

5. En effet, si le contrôle de l'appareil étatique permet à une minorité dominante de monopoliser l'allocation des ressources de la société (Burkey, 1978 : 2), l'argument culturel qui lui permet de légitimer ce monopole est généralement la revendication de l'antériorité de peuplement (Kaufmann, $2004: 4$ ).

6. Le Traité de Capitulation du 3 décembre 1810 qui cède l'île à la Grande-Bretagne stipule que les propriétés, religion, lois et coutumes des habitants seront respectées. Ce qui explique qu'ultérieurement, toute atteinte à ces points du Traité sera considérée par les FrancoMauriciens comme violation de droits imprescriptibles. Dans le temps, un glissement s'effectue de la notion juridique de défense des droits à l'argument identitaire de la défense des marqueurs ethniques menacés, expression d'une identité obsidionale.

7. Le débat sur la Rétrocession survient dans le contexte des débuts de la démocratie représentative à Maurice. La Constitution de 1885, en introduisant le principe semi-électif pour désigner une partie des membres de l'assemblée législative, qui étaient auparavant majoritairement franco-mauriciens, pose pour ces derniers la question du partage du pouvoir politique avec les autres groupes ethniques. En vertu du suffrage censitaire, une partie de la population de couleur accède au droit de vote, tandis qu'une large majorité de la population indienne en est encore exclue.

8. Pour justifier un retour de l'île Maurice à la France, les Rétrocessionnistes se réclament d'une législation antérieure à la Capitulation de 1810, l'Ordonnance Royale de 1642 qui consacrait à perpétuité la qualité de naturels français aux colons de l'île Maurice (alors Isle de France), mais avait été abrogée de fait par la capitulation.

9. Jusque dans les années 1960, Franco-Mauriciens et Hindous sont très peu en contact dans la vie quotidienne et c'est surtout à travers le processus politique que de nouvelles relations commencent à se développer entre les deux groupes (Simmons, 1982: 104). Avec l'explosion démographique qui se produit à partir de 1947, l'effet de condensation propre à l'espace insulaire rend plus aigu l'enjeu du maintien des distances ethno-sociales, alors que ne se sont pas encore mis en place les mécanismes de «communication de la différence culturelle » (Eriksen, 1993 : 27) entre les deux groupes. 
10. Ce chiffre approximatif résulte d'un calcul par soldes et ne prend pas en compte les données de l'accroissement naturel. En l'absence de statistiques officielles sur la composition du groupe blanc après cette date, ces chiffres fournissent une estimation.

11. L'émigration officielle (qui ne représenterait toutefois que $32 \%$ du mouvement total migratoire, lequel reste donc largement clandestin), toutes directions confondues, concerne un peu plus de 13170 personnes pour la seule période 1965-68, où le mouvement atteint son apogée. Les Franco-Mauriciens ont largement anticipé le changement constitutionnel, si bien que leur mouvement migratoire est pratiquement terminé dans les années soixante, tandis que l'exode se poursuit parmi les autres groupes. De 1961 à 1973, un total de 33749 émigrants, toutes destinations confondues, quittent officiellement Maurice (Boudet, 2004 : 304-5).

12. Ainsi, on a pu noter une rémigration de l'Afrique du Sud vers Maurice à chaque retour au pouvoir $(1982,2000)$ du Mouvement Militant Mauricien, dont le leader est considéré comme un Franco-Mauricien, même s'il n'est pas issu de l'élite sucrière (Boudet, 2004 : 374).

13. La métaphore de la diaspora pollinisée telle que nous l'entendons se distingue de la façon dont elle a été utilisée par Robin Cohen. Robin Cohen considère que les diasporas hybrides fonctionnent sur le schéma de la pollinisation croisée, qui dans cette conception fait référence à une propagation par vagues, y compris par l'air, au sens de diffusion des idées et de la culture (Cohen, 1997 : 178). Dans notre approche, elle évoque une dispersion qui s'effectue en conservant des caractères propres à l'individu d'origine, tout en prenant en compte la possibilité d'évolution et de différenciation lorsque la fécondation qui en découle est croisée.

14. À cet égard, la logique de la pollinisation se distingue du métissage, de la créolisation et de l'hybridation, notions qui impliquent toutes une distance préalable à l'union, une hétérogénéité ou une différence initiales.

\section{RÉSUMÉS}

Cet article examine le passage en diaspora de la minorité anciennement dominante des FrancoMauriciens (ou Mauriciens blancs d'origine française), qui au moment de la décolonisation de Maurice (1947-1968) ont émigré massivement, vers l'Afrique du Sud en particulier. La mémoire diasporique qui s'est constituée sous l'impulsion de la peur d'un "péril hindou ", revêt un caractère à la fois obsidional et dominant et légitime l'attachement de la diaspora à la nation mauricienne en le fondant sur la revendication du caractère de primo-arrivants dans l'île. La dispersion des Franco-mauriciens ne relève pas d'une logique unitaire, fonctionnant sur le mode de la filiation comme les diasporas classiques, sans pour autant se satisfaire d'une conception rattachant le groupe à une culture nomade suivant le modèle du rhizome. La métaphore de la pollinisation ouvre une troisième voie dans l'étude des diasporas, évoquant une dispersion qui cherche à se perpétuer sur le mode du semblable, du proche.

The Franco-Mauritians: a Pollinized Diaspora. This paper deals with the diasporization of a formerly dominant minority in Mauritius, the Franco-Mauritians (or white Mauritians of French descent) who massively migrated mainly to South Africa during the decolonization of the island between 1947 and 1968. The fear for a "hindu menace" which triggered their emigration shaped a diasporic memory articulated around the preservation of their identity against assimilation and a continued sense of belonging to the nation based on the claim for being the founders on the island. We argue that the dispersion of the Franco-Mauritians neither corresponds to the 
classical diaspora articulated around the idea of root and filiation, nor to the hybridity model of travelling cultures and random multiplication of references. The metaphor of pollinization rather refers to a dynamic of dissemination towards what seems similar and accessible.

Los Franco-Mauricianos: una diaspora polinizada. Este artículo trata de la diasporización de una minoría antaño dominante en la isla de Mauricio, los Franco-Mauricianos (o Mauricianos de origen francesa) que durante la descolonización de la isla entre 1947 y 1968 emigraron principalmente a África del Sur. El miedo a una "amenaza hindú" que impulsó la emigración ha fraguado en una memoria diaspórica de minoría amenazada por la asimilación cuya relación a la nación mauriciana aun perdura legitimándose con el argumento de haber sido los fundadores de la sociedad insular. Defendemos que la diaspora franco-mauriciana no se corresponde con la diáspora clásica, articulada alrededor de la idea de filiación y raíz, ni tampoco al modelo híbrido de las culturas nómadas, sino a un tercer modelo de la "diaspora polinizada". La metáfora de la polinización se refiere más bien a dínamicas de diseminación a travès de lo que parece próximo y accesible.

\section{AUTEUR}

\section{CATHERINE BOUDET}

Chargée de cours en Sociologie, Chercheur-associé au laboratoire Oracle, Université de La Réunion, 15 avenue René Cassin, BP 7151, 97715 Saint-Denis cedex 9, La Réunion. E-mail : catherine.boudet@univ-reunion.fr 